fluid therapy (NRFT) in patients undergoing laparoscopic and open colorectal surgery.

Methods A simple model was applied to evaluate the various variables reported in the published randomised, controlled trials comparing the role of RFT and NRFT by the use of principles of meta-analysis. The primary outcome measure was postoperative morbidity. Secondary endpoints were mortality and hospital stay. A random effects model was applied.

Results Seventeen randomised, controlled trials on 2165 patients were included. The incidence of postoperative morbidity (OR 0.84; $95 \% \mathrm{CI} 0.57$ to $1.24 ; \mathrm{z}=0.90 ;=0.37$ ) and mortality (OR $0.93 ; 95 \% \mathrm{CI}$ 0.47 to $1.84 ; z=0.20 ;=0.84$ ) was statistically similar following the use of either RFT or NRFT. In addition, both techniques of fluid therapy were associated with similar length of hospital stay (standardised mean difference, $-0.12 ; 95 \%$ CI -0.55 to 0.31 ; $\mathrm{z}=0.53 ; \mathrm{p}=0.59$ ).

Conclusion This meta-analysis suggests that RFT in patients undergoing laparoscopic and open colorectal surgery does not offer any advantage over NRFT.

Competing interests None declared.

\section{PWE-096 THE INVESTIGATION AND MANAGEMENT OF COLOVESICAL FISTULAE IN THE MODERN ERA-A SINGLE INSTITUTIONS 12-YEAR EXPERIENCE}

doi:10.1136/gutjnl-2012-302514d.96

N Ladwa, ${ }^{*}$ M Sajid, M McFall, A Miles, P Sains, M K Baig. Department of General and Laparoscopic Colorectal Surgery, Worthing Hospital, Worthing, UK

Introduction This aim of this study is to review how investigation and management of colovesical fistulae has progressed in our institution over a 12-year period and to propose a clear protocol to ensure prompt diagnosis and treatment in the future.

Methods A retrospective case note review was conducted of all patients with colovesical fistulae who underwent definitive surgery over a 12-year period. Variables collected include patient demographics, symptoms, investigations, operative data, histology, complications and length of stay.

Results 56 patients (38 male) underwent operative intervention for colovesical fistula. The most common symptoms are pneumaturia $(69 \%)$, faecaluria $(32 \%)$ and symptoms associated with recurrent UTIs $(68 \%)$. Cystoscopy was the most accurate test to identify fistulae (91\%) followed by CT (60\%) and barium enema (31\%). Two patients were unfit for major surgery and underwent palliative loop colostomies. The most common pathology was diverticular disease. Of the 54 remaining patients, $45 \%$ underwent laparoscopic resection with a conversion rate of $33 \%$ (due to adhesions or multiple abscesses). Sigmoid colectomy, (52\%) anterior resection (30\%) and hartmanns $(9 \%)$ are the most common procedures performed. Bladder repair was required in $25 \%$ of cases with a further $16 \%$ requiring partial resection. All patients received a postoperative cystogram to ensure bladder had healed and $70 \%$ of patients were defunctioned to protect the anastomosis. There was no mortality reported peri-operatively; the anastomotic leak rate was $5 \%$ and recurrence rate was $5 \%$. Median postoperative stay was 12.5 days (range 4-91) in the laparoscopic group and 16 days (range 6-62) in the open group.

Conclusion Surgical management for colovesical fistulae is effective and safe. Laparoscopic resections are increasing in popularity and deliver encouraging results comparable to open resection. A large multi-centre randomised controlled trial is required to validate its potential benefits over open surgery.

Competing interests None declared.

\section{PWE-097 CLASSIFICATION OF PSEUDOMYXOMA PERITONEI AS LOW OR HIGH GRADE ACCORDING TO THE WHO CRITERIA IS PROGNOSTICALLY SIGNIFICANT}

doi:10.1136/gutjnl-2012-302514d.97

${ }^{1} \mathrm{~N}$ Carr, ${ }^{*}{ }^{2} \mathrm{~B}$ Moran, ${ }^{2} \mathrm{~T}$ Cecil, ${ }^{2} \mathrm{~K}$ Chandrakumaran, ${ }^{3} \mathrm{C}$ llesley, ${ }^{4} \mathrm{~A}$ Mirnezami, ${ }^{2} \mathrm{~F}$ Mohamed. ${ }^{1}$ Faculty of Medicine, University of Southampton, Southampton, UK; ${ }^{2}$ Pseudomyxoma Centre, Basingstoke and North Hampshire NHS Foundation Trust, Basingstoke, UK; ${ }^{3}$ Histopathology, Basingstoke and North Hampshire NHS Foundation Trust, Basingstoke, UK; ${ }^{4}$ Department of Surgery, University Hospitals Southampton, Southampton, UK

Introduction The current WHO classification of tumours of the digestive system divides pseudomyxoma peritonei into two grades, namely low-grade and high-grade. This study was designed to correlate survival with low-grade and high-grade pseudomyxoma peritonei classified according to the WHO criteria.

Methods The histological slides of 274 consecutive patients were reviewed and designated as either low-grade or high-grade. The patients had been referred for cytoreductive surgery. The grade of the pseudomyxoma was correlated with survival data using the Kaplan-Meier method with the log-rank (Mantel-Cox) test.

Results 238 (87\%) patients had low-grade lesions and 36 (13\%) had high-grade lesions. The most common primary tumour was a low grade appendiceal mucinous neoplasm (231 cases, 84\%). Seven patients who died within 30 days of their operation (a postoperative mortality of $2.6 \%$ ) were excluded from survival analysis. Another patient was excluded because of incomplete survival data. The remaining 266 patients showed an overall 5-year survival of $63 \%$ in patients with low-grade pseudomyxoma peritonei and $23 \%$ in patients with high-grade pseudomyxoma peritonei $(p<0.001)$. Complete cytoreduction was achieved in 165 (60\%) patients; the 5year survival for low-grade and high-grade was $84 \%$ and $48 \%$ respectively in this group $(p<0.001)$. The median survival of patients who had complete cytoreduction was 7.7 years for lowgrade and 2.8 years for high-grade $(\mathrm{p}<0.001)$.

Conclusion Histological classification of pseudomyxoma peritonei as low-grade or high-grade correlates with prognosis. This may identify a group who could benefit from further adjuvant therapy which is not generally advocated for appendiceal mucinous tumours.

Competing interests None declared.

\section{PWE-098 CLOSTRIDIUM DIFFICILE DIARRHOEA-THE CHANGING HOSPITAL EPIDEMIOLOGY AND CLINICAL OUTCOMES FROM A HIGH PREVALENCE AREA IN NORTH EAST ENGLAND}

doi:10.1136/gutjnl-2012-302514d.98

${ }^{1} \mathrm{~J}$ Ross, ${ }^{1} \mathrm{P}$ Brown, ${ }^{*} \mathrm{C}$ Aldridge, ${ }^{2} \mathrm{~L}$ Lim, ${ }^{2} \mathrm{D}$ Nayar, ${ }^{3} \mathrm{~J}$ Sloss, ${ }^{2} \mathrm{D}$ Allison, ${ }^{1} \mathrm{~A}$ Dhar. ${ }^{1}$ Department of Gastroenterology, County Durham \& Darlington NHS Foundation Trust, Bishop Auckland, UK; ${ }^{2}$ Department of Microbiology, County Durham \& Darlington NHS Foundation Trust, Durham, UK; ${ }^{3}$ Department of Microbiology, County Durham \& Darlington NHS Foundation Trust, Darlington, UK

Introduction Clostridium difficile associated diarrhoea (CDAD) is an important hospital acquired infection. In 2008-2009 Co. Durham had one of the highest reported annual incidence of CDAD with 232 cases, 74.4 cases $/ 100000$ bed days. Following strict antibiotic stewardship in 2009, we set out to examine the changes to the hospital based epidemiology of CDAD in our three hospitals over a 12-month period.

Methods Between June 2010 and May 2011, 70 patients with positive stool $C$ difficile toxin were identified from the Microbiology database, and 56 case notes reviewed. Patient demographics, clinical symptoms, risk factors, severity, treatment for CDAD, and multidisciplinary team decisions were recorded. Clinical outcomes 